\title{
Multi-Level Search Algorithm for Roundness Error Evaluation by the Minimum Zone Circle Method
}

\author{
Li HUO ${ }^{a}$, Yuan WANG ${ }^{b}$, Chunyu ZHAO $^{c}$ and Ying DONG \\ Baicheng Ordnance Test Center of China, Baicheng 137001, China \\ a huoli1979@126.com, bwangyuan198110@126.com, ${ }^{c}$ iamyunitongzai@163.com, \\ bongying7711@126.com
}

\section{Keywords: Roundness, Error evaluation, Minimum zone circle, Multi-Level search}

Abstract. To rapidly and accurately evaluate minimum zone roundness error, a multi-level search algorithm (MLSA) was proposed. The algorithm at the premise of a fixed amount search point of each layer, by narrowing the length of the search area to narrow the search step until the required accuracy so far, in order to achieve a refined search. Optimize the number of each layer search mesh points to improve search efficiency. The center of all the measured points is the center of the initial layer search region, and the corresponding roundness error is the side of the initial layer research region. In the search process, take step size in search as the termination conditions, and by zone parallel translation jump out of local near optimized point. Examples of the three groups showed, assessed precision in the millisecond-level computation time can be achieved $10^{-15} \mathrm{~mm}$. MLSA for evaluating the minimum zone roundness error with higher precision, faster speed, good stability, simple and easy to implement, suitable for rapid evaluation of high-precision roundness error.

\section{Introduction}

Roundness is one of the most basic geometric forms expected from circular features. There are four common methods to evaluate roundness error, they are: least square circle (LSC) method, minimum zone circle (MZC) method, minimum circumscribed circle (MCC) method and maximum inscribed circle (MIC) method. Among them, only MZC method conforms to define, but it will be hard to solve due to the nonlinear problem. Generally all adopt different kinds of optimistic algorithm [1-7] search least global optimum point (following short "optimum point") of roundness error. Most of these algorithms are tedious and not intuitionistic. Computed result has certain of request to variational initial value scope, rate of convergence and error control is intricacies, especially when the more measuring point number, the more waste time to solve roundness error value, so is difficult to practical apply.

Owing to the objective function of roundness error minimum zone circle method is continuous monopole value function $[8,9]$. Thereby can seek "optimum point" by way of searching and realize roundness error assessment. Huang and Zheng [10] proposed regional searching (RS) method and Lei et al [11] proposed mesh search algorithm (MSA) method both divide target search region into mesh point, search point on mesh and found the least mesh point of roundness error as "optimum point". Method of Literature [10] only search one time for the target area, and wish to improve search precision by expanding the search region or decreasing step size in search, but it undoubtedly can add calculated amount remarkably. Literature [11] put forward the re-searching method on the foundation of least square result. It indeed can improve precision early, yet convergence result is not obvious when a search result approach "optimum point" lately which precision is hard to proceed with the improvement.

This paper realize rapidity and effectiveness of algorithm from following several aspect: 1) take sole point of computer can rapid resolving for initial point; 2) optimize mesh point quantity of each layer search, made search efficiency as high as possible; 3 ) take search step size as termination condition in order to keep shrunken speed of search region; 4) in consideration of may sink into local near optimized point in the search process, and put forward relevant solution. 


\section{Multi-Level Search Algorithm}

\section{The Settings of the Initial Layer Research Area}

Set search region for square. For easy of computer resolve, This paper take the center $(\bar{x}, \bar{y})$ of all the measured points as the center $O_{1}\left(a_{1}, b_{1}\right)$ of the initial layer research area, and take the roundness errors $f_{0}$ as the side $d_{1}$ of the initial layer research region, the roundness errors that use measuring point hub for the center of circle, there are $a_{1}=\bar{x}, b_{1}=\bar{y}, d_{1}=f_{0}$.

Suppose $\left(x_{i}, y_{i}\right)(i=1,2, \mathrm{~L}, n ; n>3)$ as the coordinates of measuring point, and work out the coordinates of measuring point center according to Eq. 1 .

$$
\left\{\begin{array}{l}
\bar{x}=\sum_{i=1}^{n} x_{i} / n \\
\bar{y}=\sum_{i=1}^{n} y_{i} / n
\end{array}\right.
$$

Follow Eq. 2 figure out roundness error when use measuring point hub for the center of circle.

$$
f_{0}=\max \left\{\sqrt{\left(x_{i}-\bar{x}\right)^{2}+\left(y_{i}-\bar{y}\right)^{2}}\right\}-\min \left\{\sqrt{\left(x_{i}-\bar{x}\right)^{2}+\left(y_{i}-\bar{y}\right)^{2}}\right\}
$$

\section{Optimize Choice of Each Layer Mesh Point Quantity}

On the premise that each layer mesh point amount is fixed, and take corresponding side reduction of average each mesh point is a maximum as optimize choose the condition.

Divide every side of each layer search region into $\mathrm{N}$ equal parts, then each side has $(N+1)$ equal parts, and every two links constitute $(N+1) \times(N+1)$ mesh point. Take area side of back tier search as front layer step size in search 2 times, namely new search region side shrunk as $2 / \mathrm{N}$ of previous one, shrunk magnitude as $(1-2 / N)$, whereas need the number of searching mesh point is $(N+1)^{2}$, wherefore calculate side reduction $\chi$ e of average each mesh point according to Eq. 3 .

$$
\chi_{e}=\frac{1-2 / N}{(N+1)^{2}} \times 100 \%
$$

It is known by Eq. 3, $\chi_{e}$ continues to decrease with the increase of $N$. When take $N$ as 3 and 4, $\chi_{e}$ is $2.08 \%$ and $2 \%$ respectively, shrunk effect separately is optima and second-best. But when take $N$ as an odd number, area center of different search layer change frequently, and its search effect is no better than take $N$ as neighbor even number. Therefore this article takes $N$ at 4 to proceed with grid partition.

\section{Classification of Mesh Point}

According to Eq. 4 work out all coordinates $\left(x_{m j}, y_{m k}\right)$ of mesh point when searching the $\mathrm{m}(\mathrm{m} \geqslant$ 1) layer.

$$
\left\{\begin{array}{l}
x_{m j}=a_{m}+j d_{m} / 4 \\
y_{m k}=b_{m}+k d_{m} / 4
\end{array}\right.
$$

In the equation, $j=-2,-1,0,1,2 ; k=-2,-1,0,1,2$.

Mesh point lie in the search region boundary when $j= \pm 2$ or $k= \pm 2$, and this paper calls these points "boundary points", other mesh points call " internal points", as showed in Fig.1. 


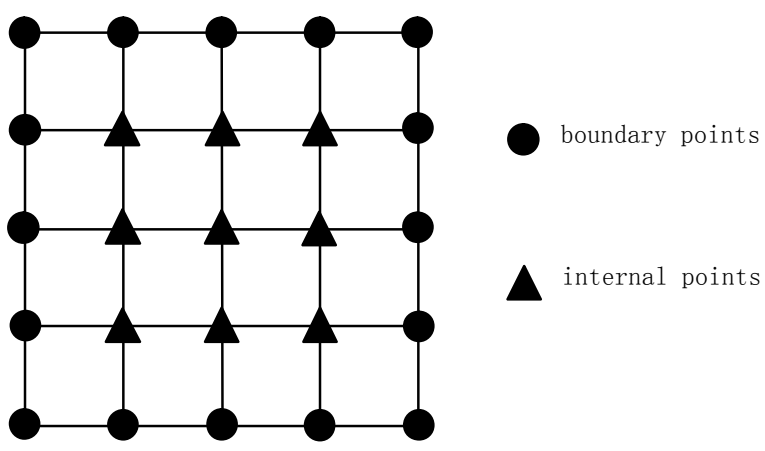

Fig.1. Classification schematic diagram of mesh point.

\section{Mesh Point Search of Each Layer Least Roundness Error}

According to Eq. 5 to calculate the roundness error $f\left(x_{m j}, y_{m k}\right)$ of taking each mesh point $\left(x_{m j}, y_{m k}\right)$ as the center of the circle when search m-th layer.

$$
f\left(x_{m j}, y_{m k}\right)=\max \left\{\sqrt{\left(x_{i}-x_{m j}\right)^{2}+\left(y_{i}-y_{m k}\right)^{2}}\right\}-\min \left\{\sqrt{\left(x_{i}-x_{m j}\right)^{2}+\left(y_{i}-y_{m k}\right)^{2}}\right\}
$$

Satisfy the condition of Eq. 6 namely is the least roundness error mesh point which gets by searching the m-th layer, and write down as $O_{m}\left(a_{m}, b_{m}\right)$.

$$
f_{m}=\min \left\{f\left(x_{m j}, y_{m k}\right)\right\}
$$

\section{Ascertainment of New Search Region}

When the least roundness error mesh point $O_{m}\left(a_{m}, b_{m}\right)$ which gets by searching the m-th layer is "internal point" in Fig.1, the "optimum point" doesn't exceed search regional scope of the m-th layer, and at the point $O_{m}\left(a_{m}, b_{m}\right)$ nearby. Now take the point $O_{m}\left(a_{m}, b_{m}\right)$ as the $(\mathrm{m}+1)$ layer of regional center in searching, and shrunk search region side. (Side of new search region is 2 times than previous step size in search.)

When the least roundness error mesh point $O_{m}\left(a_{m}, b_{m}\right)$ which gets by searching the m-th layer is "boundary point" in Fig.1, the "optimum point" may exceed this layer search regional scope. Here, if directly shrunk search region, then may omit the "optimum point". When this kind of condition appears, this paper adopts the strategy "region translation" to skip local near optimized point: take the point $O_{m}\left(a_{m}, b_{m}\right)$ as the $(\mathrm{m}+1)$ layer searching regional center, and keep the search region side magnitude unchanged.

\section{The Step of Multi-level Grid Search Algorithm}

Step 1 Follow Eq. 1 calculate the measured point center of all measured points, and according to Eq. 2 calculate the roundness error of taking measuring point center as center of the circle respectively.

Step 2 Cut various lengths of side of waiting for the search region into quarters, then have 5 division points on each side, and every two links constitute $5 \times 5$ mesh points, according to Eq. 4 calculate the coordinates of various mesh points.

Step 3 According to Eq. 5 calculate the roundness error when taking each mesh point as the center of the circle, and search out the least mesh point of the roundness error.

Step 4 While the mesh point of the least roundness error conforms to the "boundary point" shown in Fig.1, take the point as new search region center, and the new search region side remains stationary, then return to step 2; While the least mesh point of the roundness error is the "internal point" as showed in Fig.1, if step size is smaller than the object step size then stop, else take the point as the new search region center. Side of new search region is 2 times than previous step size in search, then return to step 2.

The algorithmic program flow diagram is shown in Fig.2. 


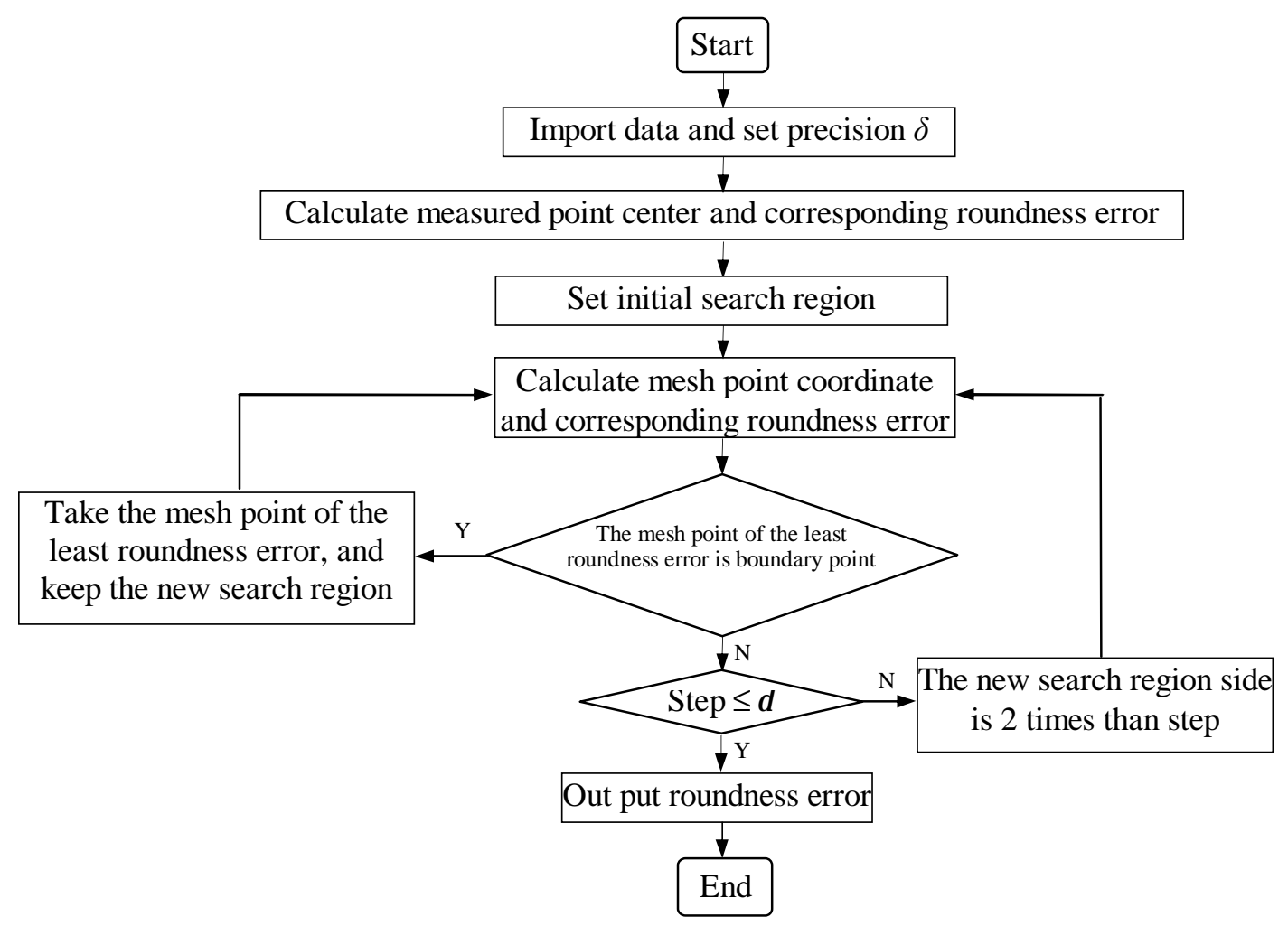

Fig.2. Flowchart of MLSA for roundness error evaluation.

\section{Experimental Results}

In order to verify the availability of the algorithm, select one typical example and two same class example of the algorithm.

\section{Dataset 1 of the roundness measurement}

The measurement dataset 1 come from literature [12], using this dataset as an example, there are part of algorithm for roundness error assessment: Genetic Algorithm(GA)[1], Effective Genetic Algorithm(EGA) [2], Fast Genetic Algorithm(FGA) [3], Particle Swarm Optimization(PSO) [4], Curvature Technique Strategy(CTS) [5], Hybrid Improved Artificial Fish Swarm Algorithm(HI-AFSA) [6], Differential Evolutionary Algorithm(DEA) [7], et al. The above algorithmic assessment results as the table 1 shows.

In this paper, firstly, put dates of the dataset 1 in Eq. 1 and work out coordinates of measuring point center are $(0.0363,-0.0531)$, then substitute Eq. 2 to figure out roundness error $f_{0}=0.0096 \mathrm{~mm}$ when take measuring point center as center of circle, which as the center of initial search region and the length of side respectively. Then conduct 9 layers searches, together search 225 mesh points, its assessment results see table 1. It can be seen from table 1 . There are good consistencies between the search result of the present algorithm and the search result of the comparison algorithm. The computation speed is superior to the comparison literatures. 
Table 1 Evaluation results of the measurement dataset 1

\begin{tabular}{|c|c|c|c|c|}
\hline \multirow{2}{*}{ Algorithm } & \multicolumn{2}{|c|}{ Central coordinate $O(a, b)$ of circle } & \multirow{2}{*}{$\begin{array}{c}\text { Roundness error } f \\
\qquad / \mathrm{mm}\end{array}$} & \multirow{2}{*}{$\begin{array}{c}\text { Time-consuming } \\
\text { /s }\end{array}$} \\
\hline & $a / \mathrm{mm}$ & $b / \mathrm{mm}$ & & \\
\hline $\mathrm{MZC}^{[12]}$ & - & - & 0.0085 & - \\
\hline $\mathrm{GA}^{[1]}$ & 0.03561 & -0.05292 & 0.0085 & - \\
\hline $\mathrm{EGA}^{[2]}$ & 0.0356 & -0.0529 & 0.0085 & 0.28 \\
\hline $\mathrm{FGA}^{[3]}$ & - & - & 0.0085 & 0.05 \\
\hline $\mathrm{PSO}^{[4]}$ & 0.03561 & -0.05311 & 0.0085 & - \\
\hline $\mathrm{CTS}^{[5]}$ & 0.0356 & -0.0529 & 0.0085 & - \\
\hline $\mathrm{HI}_{-} \mathrm{AFSA}^{[6]}$ & 0.03561 & -0.05293 & 0.0085 & 0.36 \\
\hline $\operatorname{DEA}^{[7]}$ & - & - & 0.0085 & 0.21 \\
\hline Present algorithm & 0.03561 & -0.05293 & 0.0085 & 0.0015 \\
\hline
\end{tabular}

Ref. [2] and [7] runs dataset1 20 times independently which use of EGA and DEA respectively, and standard deviation separately is $9.13 \times 10^{-7}$ and $1.14 \times 10^{-16}$. For comparison, present algorithm independently runs dataset 120 times too, and standard deviation is 0 .

There is more high precision assessment result than table 1 in Ref. [6]: center of circle (0.035 614 97, -0.052 92948 ), roundness error $f=0.008537464 \mathrm{~mm}$, take time $0.0033 \mathrm{~s}$.

For further show the present algorithm has high precision and fast speed, taking object step size $10^{-15} \mathrm{~mm}$ as to assess dataset 1 , and need carry out 44 layers search(contain 1 layer area translation search). Together search 1100 mesh points, take time $0.0073 \mathrm{~s}$, center of circle $(0.035614971220$ 512, -0.052929 481200635 ) and roundness error $f=0.008537464354593 \mathrm{~mm}$.

The above analysis fully explains that the present algorithm with higher precision, faster speed and good stability. Moreover, in contrast to algorithms in literature, the principle of the algorithm is simple and is easy to be achieved by computer.

\section{Dataset 2 of the roundness measurement}

The measurement dataset 2 takes from the roundness sampled data in Ref. [10]. The new algorithm compares with the computed result of zone search method in Ref. [10, 13], such as table 2 shows. In this paper, firstly, put data of the dataset 2 in Eq. 1 and work out coordinates of measuring point center are $(267.648,12.131)$, then substitute Eq. 2 to figure out roundness error $f_{0}=0.0117 \mathrm{~mm}$ when take measuring point center as center of circle, which as the center of initial search region and the length of side respectively. Then conduct 6 layers searches, the results as table 2 shows.

Table 2 Evaluation results of the measurement dataset 2

\begin{tabular}{|c|c|c|c|c|}
\hline \multirow{2}{*}{ Algorithm } & \multicolumn{2}{|c|}{ Center of circle coordinates $O(a, b)$} & \multirow{2}{*}{$\begin{array}{c}\text { Roundness error } f \\
\qquad / \mathrm{mm}\end{array}$} & \multirow{2}{*}{ Mesh point amount } \\
\hline & $\mathrm{a} / \mathrm{mm}$ & $\mathrm{b} / \mathrm{mm}$ & & \\
\hline Area search method $^{[10]}$ & 265.647 & 12.131 & 0.0244 & 251001 \\
\hline Area search method ${ }^{[13]}$ & 267.6484 & 12.1323 & 0.0099 & - \\
\hline Present algorithm & 267.6516 & 12.1292 & 0.0098 & 150 \\
\hline
\end{tabular}

See from table 2, the assessment result in the present algorithm excels that in Ref. [10, 13]. The need searching mesh point amount is obviously less than comparison literatures. This explains that the present algorithm with higher precision, faster speed, moreover, in contrast to algorithms in literatures, the computational process is easy to be achieved by computer automatically, without human intervention and the resolve result is unique.

Adopt the present algorithm, taking object step size $10^{-15} \mathrm{~mm}$ as to assess dataset 2, and need carry out 48 layers search (contain 5 layers area translation search). Together search 1200 mesh points, take time $0.0092 \mathrm{~s}$, center of circle (267.651 $670386421796,12.129160048368$ 146) and roundness error $f=0.009823072105661 \mathrm{~mm}$.

\section{Dataset 3 of the roundness measurement}

The measurement dataset 3 takes from the roundness sampled data in Ref. [11]. The new algorithm compares with the computed result of the MSA in Ref. [11]. In this paper, firstly, put data of the dataset 3 in Eq. 1 and work out coordinates of measuring point center are (-9.846 25, 48.770 41), then substitute Eq. 2 to figure out roundness error $f_{0}=3.8186 \mathrm{~mm}$ when take measuring point center 
as center of circle, which as the center of initial search region and the length of side respectively. Then conduct 16 layers of search (contain 16 layers area translation search), the results as table 3 shows.

Table 3 Evaluation results of the measurement dataset 3

\begin{tabular}{|c|c|c|c|c|}
\hline \multirow{2}{*}{ Algorithm } & \multicolumn{2}{|c|}{ Center of circle coordinates $O(a, b)$} & \multirow{2}{*}{$\begin{array}{c}\text { Roundness error } f \\
\qquad / \mathrm{mm}\end{array}$} & \multirow{2}{*}{ Mesh point amount } \\
\hline & $a / \mathrm{mm}$ & $\mathrm{b} / \mathrm{mm}$ & & \\
\hline MSA $^{[11]}$ & -11.4280 & 49.8497 & 0.0196 & 900 \\
\hline Present algorithm & -11.4278 & 49.8496 & 0.0193 & 400 \\
\hline
\end{tabular}

See from table 3, the assessment result in the present algorithm excels that in Ref. [11]. The need searching mesh point amount is much less than comparison literature. This illustrates that the present algorithm with higher precision and faster speed.

Taking object step size $10^{-15} \mathrm{~mm}$ as to operate dataset 3 , and need carry out 53 layers search (contain 2 layers area translation search). Together search 1325 mesh points, take time $0.0073 \mathrm{~s}$, center of circle $(-11.427810317790881,49.849585574899421)$ and roundness error $f=0.019$ $230064874986 \mathrm{~mm}$.

\section{Conclusions}

1) In this paper, we proposed the Multi-Level Search Algorithm (MLSA) which for evaluating the minimum zone roundness error with higher precision, faster speed and good stability. The evaluation results of Minimum zone method which assessment precision can be achieved $10^{-15} \mathrm{~mm}$ in the millisecond-level operation time. It is suitable for rapid evaluation of high-precision roundness error.

2) The principle of the algorithm is simple and convenient for computer programming. The assessment process needs without manual set up any of the initial condition. The computational process is easy to be achieved by computer automatically, without human intervention.

3) This algorithm has generality and preferable practicability, can expand to other assessment of form errors.

\section{References}

[1] C.C. Cui, R.S. Che, X.C. Luo, et al. Form error evaluation of circles based on a finely designed genetic algorithm. Chinese Journal of Mechanical Engineering. Vol.17(2004) No.1,p.59-62.

[2] X.L. Wen, Q.G. Xia, Y.B. Zhao. An effective genetic algorithm for circularity error unified evaluation. International Journal of Machine Tools \& Manufacture. Vol.46(2006) No.14,p. 1770-1777.

[3] A. Rossi, M. Antonetti, M. Barloscio, et al. Fast genetic algorithm for roundness evaluation by the minimum zone tolerance (MZT) method. Measurement. Vol.44(2011) No.7, p.1243-1252.

[4] C.C. Cui, F.G. Huang, R.C. Zhang, et al. Roundness error evaluation based on the particle swarm optimization. Acta Metrologica Sinica. Vol.27(2006) No.4,p.317-320.

[5] X.M.Li and Z.Y.Shi. Evaluation of roundness error from coordinate data using curvature technique. Measurement. Vol.43(2010) No.2,p.164-168.

[6] M. Ye, D.B. Tang, Z.P. Zhao, et al. Evaluation of circularity error based on hybrid improved artificial fish swarm and geometric algorithm. Journal of Nanjing University of Aeronautics \& Astronautics. Vol.45(2013)No.4,p.526-531.

[7] D.X. Wang, A.G. Song and X.L. Wen. Application of differential evolutionary algorithm for evaluation of roundness error. Acta Metrologica Sinica. Vol.36(2015) No.2,p123-127.

[8] P. Liu. Study on the convex and concave character of roundness error objective function. Acta Metrologica Sinica. Vol. 24(2003) No.2,p.85-87. 
[9] P. Liu and L.J. Du. Study on the properties of the objective function for assessing roundness errors. Journal of Astronautic Metrology and Measurement. Vol.27(2007) No.2,p.1-3.

[10] F.G. Huang and Y.J. Zheng. A method for roundness error evaluation based on area hunting. Acta Metrologica Sinica. Vol. 29(2008):No.2,p.117-119.

[11] X.Q. Lei, W.H. Chang, Y.J. Xue, et al. Evaluation of roundness error based on mesh search algorithm. Chinese Journal of Scientific Instrument.Vol.29(2008) No.11,p.2324-2329.

[12] W.Y. Jywe, C.H. Liu and C.K. Chen. The min-max problem for evaluation the form error of a circle. Measurement. Vol.26(1999) No.4,273-282.

[13] F.G. Huang and Z.P. Dong. Linearization method of roundness error evaluation based on principle of measuring coordinates. Journal of Huaqiao University (Natural Science). Vol.32(2011) No.5,p.492-494. 